\title{
Developing online approaches to visually inspect quality characteristics with known tolerances
}

\section{Mohammed Hussein*}

Mechanical Engineering Department, British University, P.O. Box 43, Al-Shorouk City 11837, Egypt

Email: mohammed.hussein@bue.edu.eg

${ }^{*}$ Corresponding author

\section{Safaa Labib Diab}

Department of Physics,

Helwan University,

Helwan, Egypt

Email: safaa_labib1961@yahoo.com

\begin{abstract}
In this work, new approaches for online 100\% visual inspection are introduced. Unlike the traditional visual inspection approaches that testing the complete similarity of the inspected unit with a master one, the developed approaches test whether each quality characteristic in the product falls within its specified tolerance band. In the first approach, some statistical indices were used, e.g., correlation, to decide whether the dimensions of the inspected product are within specs. This approach is then modified, and each key dimension is treated alone to know which one (if any) falls beyond its tolerance zone. A third approach is introduced in which a feature of each quality characteristic is developed, and a $\mathrm{NN}$ model is used to determine whether this quality characteristic comply with its specs. The decision is released in the real time such that an out of control situation can be treated immediately.
\end{abstract}

Keywords: visual inspection; tolerance bands; neural network; image processing; feature extraction.

Reference to this paper should be made as follows: Hussein, M. and Diab, S.L. (2015) 'Developing online approaches to visually inspect quality characteristics with known tolerances', Int. J. Collaborative Enterprise, Vol. 5, Nos. 1/2, pp.19-31.

Biographical notes: Mohammed Hussein is a Professor of IE in the Mechanical Engineering Department, IE Division, Faculty of Engineering, Helwan University, Cairo, Egypt. He is currently on leave to the British University in Egypt (BUE). He received his PhD from a joint research between California State Poly Technical University and Helwan University. His research scope includes quality control, technology transfer and material management. 
Safaa L. Diab is an Assistant Professor in the Faculty of Science, Helwan University, Cairo, Egypt. She received her $\mathrm{PhD}$ from a joint research between University of Dayton, Ohio, USA, and Helwan University. Her research interests include different applications of image processing.

\section{Introduction}

The challenges facing manufacturers currently include aggressive competition to produce high-quality products with nearly zero defects at reasonable costs. The traditional statistical quality control methods sometimes do not prevent producing defective products completely unless the capability indices of the production processes are relatively high. Most of the pioneering manufacturing firms turn to $100 \%$ inspection policy to make sure of not allowing defective products to pass to the customers. However, the high costs and long inspection time of such policy sometimes limit the use of this approach.

Existing methods of inspection are often inadequate and are the main limitation to improvement. Automated visual inspection represents a significant contribution to the second industrial revolution. Automated visual inspection is taken to encompass a wide variety of tasks, including blemish detection, complex patterned-surface examination, single and multiple defect detection, measurement of tools and components, counting, grading, sorting, sizing, orientation and posture recognition (for robot control), in process monitoring and control. Visual inspection can also be extended to cover other situations where images are generated by thermography, radiography, fluoroscopy, NMR and ultrasonic. These are classified as non-imaging uses of light. Visual inspection is used currently in many industrial applications, e.g., printing circuits, ceramic paints, printing, converting, packing and pharmaceutical industries. There are many commercial software packages that are used in visual inspection like 'VisionView', 'Cognex in-sight', and 'Gardian PQV'. In all of these applications, the inspection process is used to check that the produced units are copies of their master product. The inspection result is either to accept in case of coincidence or to reject otherwise. No further investigation about the defect location or size, or if there is a possibility to rework a defective product. In mechanical machining processes, dimensions of products' quality characteristics are not necessarily coincide with their nominal values; but they should fall within their design tolerance bands. This makes the traditional visual inspection methods not suitable for this application.

In this research, the authors introduce some approaches to overcome this problem; and accept products with dimensions that have within-tolerance deviations from their nominal values. These approaches integrate image processing techniques and statistical indices to release inspection results. All the required tools to apply these approaches are a digital camera fixed in the production line, a PC and the coded computer package that performs image processing and releases inspection results.

\section{Previous work}

Automated visual inspection is a task that proves its valuable technical and economical benefits. It is efficiently used in many applications (Newman and Jain, 1995), e.g. 
medical (Kazdin, 2011; Sankaranarayanan et al., 2003), pharmaceutical and industrial (Hussein and Diab, 2010a, 2010b). Accordingly, a lot of commercial systems have been developed and utilised in different applications (http://www.cognex.com/visualinspection-systems-visionview.aspx; http://www.cognex.com/support/insight/). In this survey, the concentration is on industrial vision applications.

The industrial applications of vision systems can be classified into several areas, namely: dimensional quality, surface quality, materials microstructure tests, correct assembling and operational process quality (Davies, 1998).

Inspection of dimensional defects has become a critical task for manufacturers who strive to improve product quality and production efficiency (Lee and Vachtsevanos, 2002; Lin, 2008). Research concerning automated visual inspection grows rapidly. Leopold et al. (2003) introduced new approaches in fast 3D-surface quality control. They made a good classification of these approaches and their application fields.

Checking whether the dimensions of a product are within specified tolerances and the products have the correct shapes is ordinary tasks for industrial quality control systems. Such tasks involve characteristics of products in two or three dimensions, namely, the inspection of dimensional quality (Davies, 1998).

There are several application fields where image measurements can assume a great relevance. Chen and Ventura (1995) introduced a vision-based shape recognition and analysis system of machined parts. Their work involved the development of machine vision algorithms for automated inspection of production parts. The inspection system consists of three parts in series: segmentation, recognition and analysis. The input of this system is a set of ordered boundary data extracted from the product image and the output includes the identity of this product: its pose, dimension and out-of-profile error. They used computer experiments to show that their proposed algorithms are accurate and fast.

Most of researches integrated AI approaches with image processing tools. Lin (2009) developed an approach that utilises neural network and statistical approach to inspect light-emitting diode chips. Fricout et al. (2008) developed an online approach to inspect the smoothness of metal surfaces. The measurements were based on topographical maps obtained through interferometric microscopy. The resulting data were analysed by an algorithm based on morphological and statistical features extraction from surface topography, factorial analysis, bootstrap over-sampling and Bayesian classification. Lin (2007) presented a wavelet characteristic based approach for the automated visual inspection of ripple defects in the surface barrier layer chips of ceramic capacitors.

Among the useful applications of visual inspection is the colour unevenness testing like in LCD monitors which is not easy to be made by humans as a result of human subjectivity and eye fatigue. Chiu and Lin (2009) developed a hybrid approach to test blemishes in LCD panels. Their approach is based on Hotelling statistics and image analysis.

The decision of all developed approaches is either to accept or reject the product without determining the source of $\operatorname{defect}(\mathrm{s})$. The approaches introduced in this research utilise image processing and statistical approaches to inspect simultaneously multiple quality characteristics. It is recognised by giving a decision for each quality characteristic such that a product defect(s) is recognised; and not just accept/reject decision. 


\section{The developed approaches}

The following approaches are developed to inspect quality characteristics in two dimensions. The developed visual inspection approaches use image processing techniques and some statistical indices to perform inspection. The following general steps are common in the inspection process, with some differences from one approach to another. First, an image of the master product, a product with nominal quality characteristics dimensions is to be acquired. The images of the products to be inspected are captured and threshold. Secondly, a feature is to be extracted for the threshold images. This feature depends on the approach used and will be detailed for each approach. Third, comparison between extracted features of the images and that of the master product is conducted. The comparison tells whether the product's quality dimensions are complying with the designed ones through either calculated statistical factors or a direct decision from a designed neural network.

\subsection{Statistically based visual inspection approaches}

In these approaches, the image matrix of the inspected product is converted to the greylevel (with only 0 , and 1 elements) and then threshold. The whole processed matrix is considered as the feature. Two statistical parameters were used to compare the features: the correlation factor and root mean square error (RMSE). If the calculated parameter (correlation or RMSE) falls within certain range then the product is considered acceptable. The acceptance ranges of the parameters are determined experimentally. To illustrate these steps, consider the product illustrated in Figure 1. The product consists of five quality characteristics: two small circles, two slots and one big circle. The dimensions of a product are as follows: The two small circles are $4 \mathrm{~mm}$ diameters and the big circle is $20 \mathrm{~mm}$ diameter. The two slots are identical; the length of the slot is $20 \mathrm{~mm}$ and its width is $4 \mathrm{~mm}$. The tolerance of each dimension is $\pm 0.5 \mathrm{~mm}$.

Figure 1 The reference image

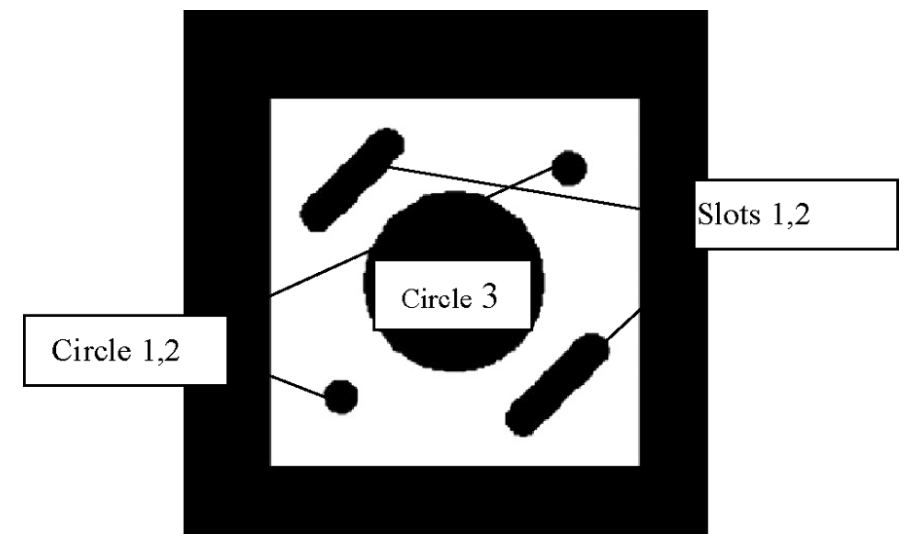

The correlation between the reference image matrix and that of an inspected product is given by equation (1). 


$$
\mathrm{Cr}=\sum \frac{(\mathrm{imw}-\mathrm{mw}) \times(\mathrm{imf}-\mathrm{mf})}{\operatorname{SQRT}\left(\sum(\mathrm{imw}-\mathrm{mw})^{2} \times \sum(\mathrm{imf}-\mathrm{mf})^{2}\right)}
$$

and the mean root square error is calculated as by equation (2).

$$
\operatorname{RMSE}=\operatorname{SQRT}\left(\sum_{x=0}^{M-1} \sum_{y=0}^{N-1}[w(x, y)-f(x, y)]^{2}\right) /(M \times N)
$$

where 'imw' and 'imf' are the matrices of the product image and the reference image (having the same size), $\mathrm{mw}$ and $\mathrm{mf}$ are the mean values of the $w$ and $f$ matrices, and $M \times N$ is the matrix size.

\subsection{Multi-index visual inspection approach}

The approaches in Section 3.1 tell whether the product dimensions are within acceptable ranges if the calculated correlation is above certain critical value or if the RMSE is below its critical value. The critical values are determined experimentally. The main shortcoming of these approaches is that they do not recognise the defect location, i.e., if there is a defected product, one cannot know which quality characteristic is defected unless traditional measurements are conducted. This shortcoming is avoided in the multiindex approach.

In this approach, each quality characteristic is convoluted with a mask as shown in Figure 2. A separate index for each quality characteristic is calculated as in equation (3).

$$
I_{m}=\sum_{i=1}^{n_{m}} \frac{s_{i}}{n_{m}} \quad m=1,2, \ldots, M
$$

where $S_{i}$ is a $(0,1)$ variable; which is one when the mask's $i$ pixel is similar to the corresponding one of the reference image and zero otherwise, $n_{m}$ is the number of pixels of mask $m$, and $M$ represents the number of masks or number of quality characteristics.

Figure 2 The reference image with masks

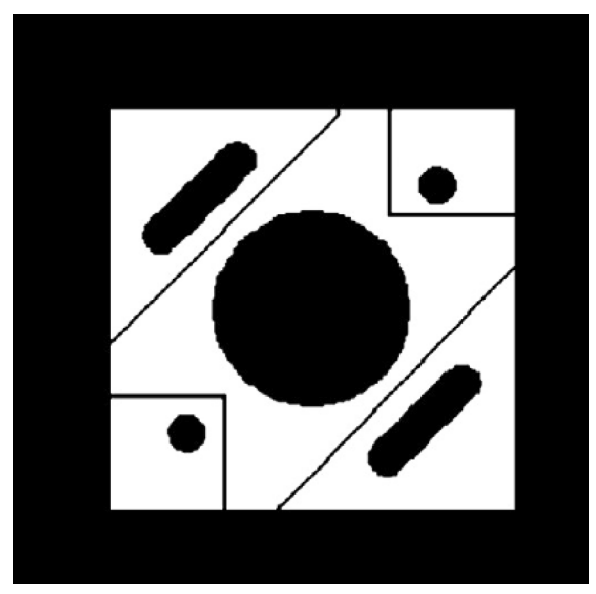


The set of indices is the extracted feature. According to the value of an index, its quality characteristic's dimension is considered accepted or rejected if this value is above or below a critical value. The critical indices values are calculated experimentally.

\subsection{Neural network (NN)-based visual inspection approach}

The NN-based inspection methodology includes image acquisition, image pre-processing, feature extraction and inspection procedures.

a Image acquisition: VP-EYE Image software is used to acquire the product image then the images are stored in the computer.

b Image pre-processing: After the acquired images have been stored, the image preprocessing algorithm is executed using the Matlab-7 software, which includes three steps:

1 Convert the image from RGB image to grey-level image.

2 A threshold process is used to convert the grey image into a binary image. The grey scale value under specified threshold is assigned to ' 0 ' and others are assigned to ' 1 '. The binary value can be expressed as by Jain (Lin, 2009) as follows:

$$
B(x, y)= \begin{cases}1 & \text { if } f(x, y) \geq k \\ 0 & \text { otherwise }\end{cases}
$$

In this research, the threshold value is calculated automatically through Matlab-7 and is equal to 0.46 .

3 Filtering: The filtering is done using morphological operations; the number of pixels added or removed from the objects in an image depends on the size and shape of the structuring element (SE) used to process the image (Gonzalez and Woods, 2002). In the morphological operations, the state of any given pixel in the output image is determined by applying a rule to the corresponding pixel and its neighbours in the input image. The morphological operations are used to remove the noise, which called the morphological filter. The filtration is done by removing all connected components (objects) that have fewer than 30 pixels using the Matlab-7 function (bwareaopen). Thus, the objects with a radius of less than 30 pixels are eliminated. Afterwards another morphological operation closing is implemented using (imclose) function to perform morphological closing on the image.

c Feature extraction: Features to be extracted for each of the quality characteristics of the product, namely, the two small circles, the big circle and the slots. Each character is divided into eight divisions. The steps for extracting these characters are image inversion and segmentation.

d Image inversion: It means inversing the binary of image value ' 0 ' and ' 1 ' into ' 1 ' and ' 0 '. It is a necessary step, because the image of the character is wanted not to be the image of background. The image inversion can be expressed as 


$$
B^{\prime}(x, y)= \begin{cases}1 & \text { if } B(x, y)=0 \\ 0 & \text { if } B(x, y)=1\end{cases}
$$

where $B(x, y)$ is the binary value and $B^{\prime}(x, y)$ is the inverted binary value.

e Segmentation: The segmentation of an image into various objects from the background is implemented. Two segmentations are done; first segmentation is executed by separating each character out from the product image. Figure 3 shows the result of the first segmentation of each character using Matlab-7 software.

Each quality characteristic is centred in a box that is dominant to its size. The boxes of the master product image (image with nominal sizes) are similar to their relevant in each inspected product image.

As the characters of the product are extracted and each character has appeared in a box (the features extracted for statistical approach), a second segmentation is executed by dividing each character into eight-equally divisions. Figure 4, for example, shows a circle centred in a box and its divisions including portion number 1 up to 8 .

Figure 3 Quality characters segmentation

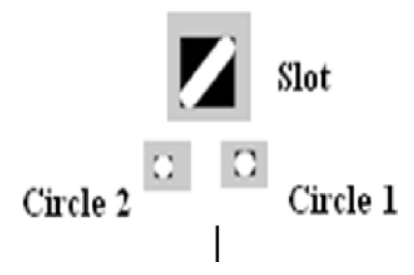

Figure 4 The divisions of circle 1

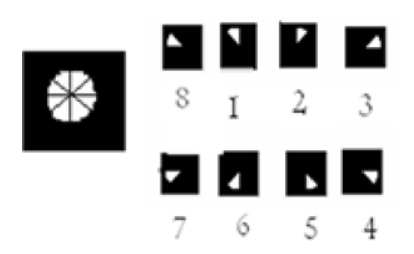

Let the centroid coordinates of any character is $(r, c)$ and the centroid of portion number one is $(\mathrm{r} 1, \mathrm{c} 1)$, for example. The feature required for neural network approach is the set distances, $d=\left\{d_{1}, d_{2}, \ldots, d_{8}\right\}$, which is the distances between the character's centroid and the centroids of the eight division. For example, the distance $d_{1}$ between the character centroid $(r, c)$ and that of the first division $\left(r_{1}, c_{1}\right)$ is calculated from equation (6) as follows:

$$
d_{1}=\sqrt{\left[\left(c_{1}-c\right)^{2}+\left(r_{1}-r\right)^{2}\right]}
$$

Figure 5 shows the centroid of the whole character and the centroid of the portion number one. For each character, there are eight-distance values, meaning that each image have 40 values of distances arranged in one vector called feature vector. 
Figure 5 The two centroids and the distance $(d)$ (see online version for colours)

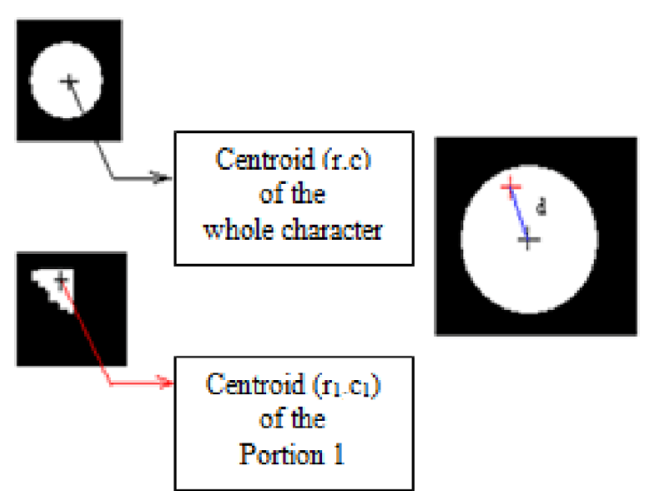

The developed $N N$ inspection model: A back propagation neural network is used as a classifier. The network structure is composed of an input layer, two hidden layers and an output layer. The number of neurons in each of the hidden layer is 40 and only one neuron in the output layer. Figure 6 shows the network architecture. The network is trained with a set of products with different dimensions. The feature vectors extracted from the product images are submitted to the neural network training.

The network is trained using the back propagation training algorithm (Zurada, 1992). Delta learning rule is applied which includes two phases. During the first phase, the input vector $x$ is introduced and propagated forward through the network to compute the output value $y$ for each output unit. This output is compared with its desired value $d_{o}$, resulting in an error signal for each output unit. The second phase involves a backward pass through the network during which the error signal is passed to each unit in the network and appropriate weight changes are calculated.

The developed network, training data and desired outputs are processed through the Matlab-7 software, which automatically trains the network and creates the error reduction progress curve during the training processes. The resulting error value is very small and very close to zero.

Figure 6 The neural network architecture

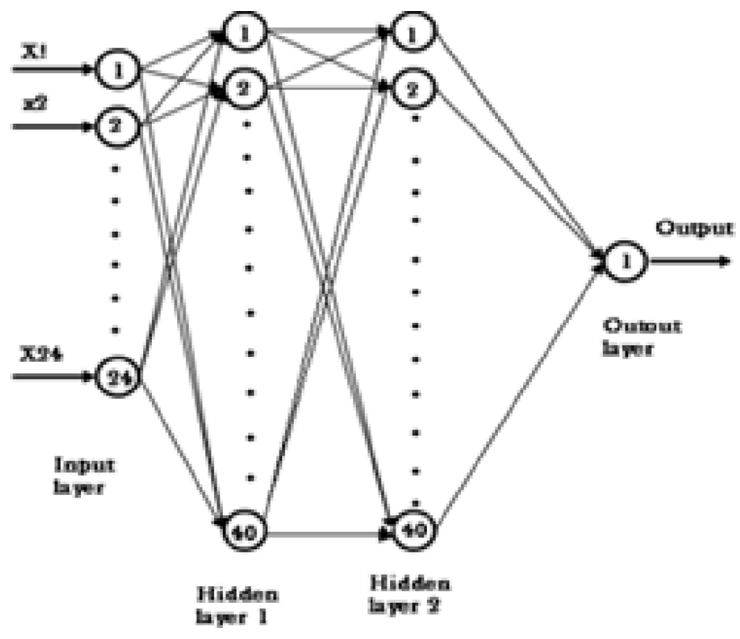




\section{Results and analysis}

\subsection{Results of the first approach}

The correlation and the RMSE are calculated for 169 product images. In a previous work (Hussein and Diab, 2010a), the relations between both of the correlation coefficient and the RMSE Vs the tolerances of the product dimensions were proved. In this context, the relationship between the first circle diameter and both $\mathrm{Cr}$ and RMSE was proved to be almost linear (blocking the rest of dimensions). USL and LSL $(4 \pm 0.5 \mathrm{~mm})$ were used to determine the critical correlation and RMSE values.

Since the product contains multiple characteristics to be inspected, the correlation coefficient and RMSE are calculated for the 169 images in which the first 121 ones are for within tolerance products (accepted products). The results are exhibited in Figures 7 and 8, respectively. From Figure 7, it can be noticed that the accepted products (the first 121 ones) have correlation coefficient values higher than those of the rejected ones. The critical correlation coefficient of the whole product (as by Figure 7) is 0.992 .

Figure 7 Correlation coefficient for all images

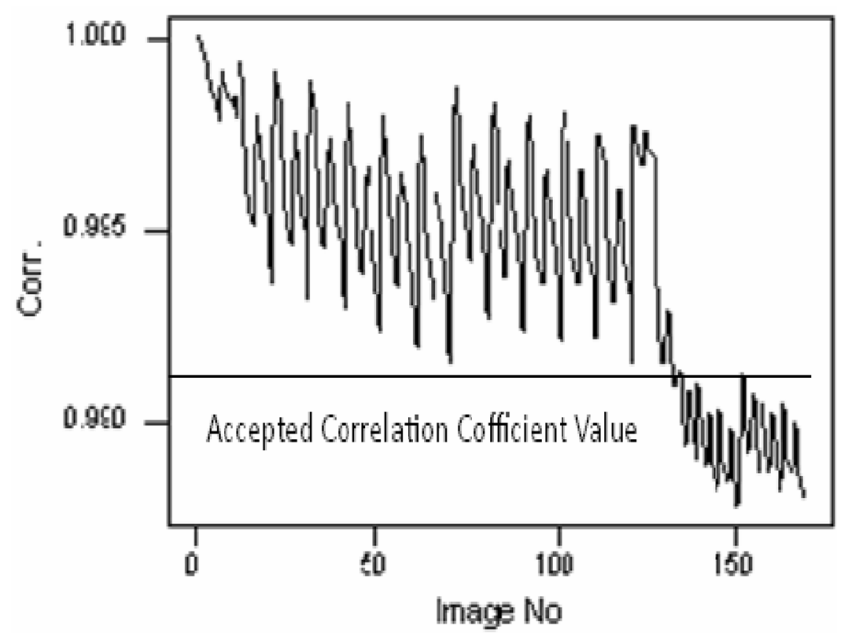

Figure 8 RMSE for all images

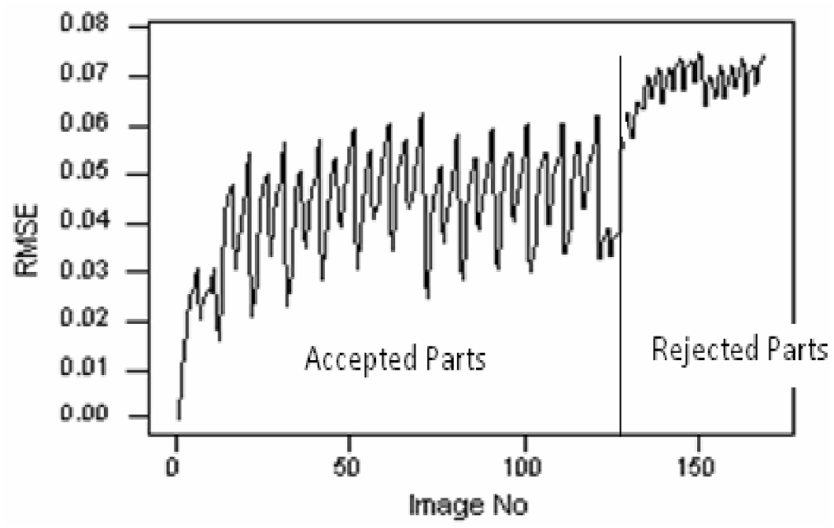


Also, Figure 8 illustrates that RMSE of the accepted products can be distinguished from those of the rejected products. Using Figure 7, one can concludes that if the correlation between a product's image and the reference one is 0.992 (or more) or if the RMSE between them is less than 0.06 , the product is considered acceptable and vice versa.

\subsection{Results of the second approach}

Applying equation (4), the indices for the five quality characteristics were calculated simultaneously. Each pixel of a product's image matrix is compared to its corresponding one of the reference image; and according to its location (the mask it belongs to), the index value of that mask is updated. The value of an index is exactly equal one (theoretically) if the quality characteristic dimensions are the same as the reference one and has no shift in position. Deviation from that target downsizes the index value. Experimentally, one can determine an acceptable value lower limit for $I_{m}$ when the dimensions of the quality characteristic fall within the tolerance region. The approach can also be used to detect a shift in the quality characteristic position. If, for example, a hole's centre is shifted from its correct position, the calculated index will go down even if the hole diameter is within tolerance.

The determination of acceptable indices' values of the quality characteristics as well as the digital image data processing requires experimental work. In this context, the authors used 169 images with different dimensions that all fall within the tolerance specifications, 71 images with some out of tolerance dimensions and 30 images with shifted quality characteristic(s) positions. The resulted quality characteristics indices of all tested images are plotted vs. the characteristics' dimensions. A regression analyses were conducted to test the relationship between a dimension deviation from the nominal size and the corresponding value of the index. As an example, the regression analysis for the slot characteristic conducted using Minitab-12 is shown in Table 1.

Table 1 Regression analysis for the slot characteristic

\begin{tabular}{|c|c|c|c|c|c|}
\hline \multicolumn{6}{|c|}{ Regression analysis $S 1$ INDEX vs. DC3 } \\
\hline \multicolumn{6}{|c|}{ The regression equation is $S 1$ INDEX $=1.00-0.0207 D C 3$} \\
\hline Predictor & Coef. & SE coef. & $T$ & $P$ & \\
\hline Constant & 0.999557 & 0.000156 & 6388.84 & 0.000 & \\
\hline DC3 & -0.0206534 & 0.0003988 & -51.79 & 0.000 & \\
\hline \multicolumn{6}{|c|}{$S=0.001608, R^{2}=91.8 \%, R^{2}($ adj. $)=91.8 \%$} \\
\hline \multicolumn{6}{|c|}{ Analysis of variance } \\
\hline Source & $D F$ & $S S$ & $M S$ & $F$ & $P$ \\
\hline Regression & 1 & 0.0069354 & 0.0069354 & 2682.21 & 0.000 \\
\hline Residual error & 239 & 0.0006180 & 0.0000026 & & \\
\hline Total & 240 & 0.0075534 & & & \\
\hline
\end{tabular}

As can be noticed, there is almost a perfect linear relationship between the slot width and the index. The ANOVA results show how strong is the linear relationship (very low values of square errors either for the constant or for the slope). The results of other characteristics sustain the same conclusions. Accordingly, such a linear relationship can 
be used to predict the actual width if the index value is known. The critical values for different characteristics' indices, detailed in a previous work (Hussein and Diab, 2010a), are given in Table 2 .

There are some advantages of this approach. First, it can tell about the compliance of each character to its tolerance specification simultaneously; accordingly, one can determine directly if there is any defect and the location of that defect(s). Second, if there is a position shift in a quality characteristic, it can be detected (Hussein and Diab, 2010a, 2010b). Third, the regression model can be used to measure the character's dimension especially linear dimensions. Finally, the approach can be helpful when the quality characteristic has a complicated shape that cannot be measured directly.

Table 2 Lower limit of inspection index

\begin{tabular}{lccc}
\hline Quality characteristic & The small hole (circle 1) & The big circle (circle 2) & The slot (slot 1) \\
\hline Index lower limit & 0.993 & 0.983 & 0.989 \\
\hline
\end{tabular}

\subsection{Results of the NN-based approach}

A back propagation neural network is used as a classifier. The network structure is composed of an input layer, two hidden layers and an output layer. The number of neurons in each of the hidden layer is 40 and only one neuron in the output layer. Figure 6 shows the network architecture. The network is trained with a set of products with different dimensions. The feature vectors extracted from the product images are submitted to train the neural network.

The developed network is trained with 280 product images and tested with additional 191 product images. The method used to extract features in the training stage is used to extract the features in the testing stage of testing images. Then testing the neural network is done. Matlab-7 displays the acceptance/rejection decision of testing images in Matlab window. If the dimension of one character in the product is out of tolerance, the product as a whole is rejected. In our approach, the acceptance/rejection decision results are based on the product as a whole not on each character. The trained neural network is then used to indicate the acceptance/rejection decision. The results show that all acceptance/ rejection decisions are completely perfect, meaning that the inspection efficiency is $100 \%$.

The developed network, training data and desired outputs are processed through the Matlab-7 software, which automatically train the network and create the error reduction progress curve during the training processes. The resulting error value is very small and very close to zero. More details can be found in previous work (Hussein and Diab, 2011; Diab et al., 2011).

Neural network gives good inspection results. The wrong decisions obtained from the statistical approaches are belonging to circle 2 . The correlation coefficient values are very close to one, and any numerical approximation may affect the acceptance/rejection decision. As the differences between the master and the inspected part's images are not enough to affect the statistical values, the solution of this problem is to enlarge the matrices of each character. Enlarging the matrices does not mean increase the resolution of the camera, but means increasing the FOV of the telecentric lens used in image capturing. 
The superior performance of the NN approach in comparison to that of the statisticsbased approaches can be a result of:

- The testing products are very close to those of the training ones, and that means if we use more testing products with significant variations, the NN results may be different. Beside, the results obtained from the NN approach do not give the quality characteristic(s) that is out of tolerance in case of rejecting a product.

- Unlike the statistical approaches, the NN approach is not sensitive for noise.

\section{Conclusions}

From the results of using different visual inspection approaches, it can be concluded that:

- Computer vision offers consistency, accuracy and repeatability, in contrast to the subjectivity, fatigue, slowness and cost associated with human inspection.

The advantages of using a machine vision system for inspection include a decrease in the time required for inspection as well as greater accuracy of inspections and better flexibility than the conventional methods.

- The developed approaches are effective in inspecting 2D machined multiplecharacteristic products at low cost. The product size to be inspected using the developed visual inspection approaches should be relatively small, up to $50 \times 50 \mathrm{~mm}$.

- The inspection approaches can be used online to segregate rejected products from production lines. The user's skill has no influence in the final result and a faster inspection has been realised in real time.

- The neural network approach gave $100 \%$ error-free inspection results that is better compared to statistical approaches.

- The problem with statistical approaches is that the number of pixels that are different between the master and the inspected images is very little compared to the total number of pixels. This gives the reason of high of accepting correlation values or low of MSE values. Accordingly, the statistical approaches are sometimes insensitive to small variations. On the other hand, the sensitivity of NN can be increased by increasing the number of neurons of the hidden layers.

- The methodology of feature extraction utilised in training and testing the NN makes comparisons for low number of pixels in which differences exist while most of the pixels are identical. Accordingly, NN needs big number of images in order to be trained.

\section{References}

Chen, J.M. and Ventura, J.A. (1995) 'Vision-based shape recognition and analysis system of machined parts', International Journal of Production Research, Vol. 33, No. 1, p.101. 
Chiu, Y.P. and Lin, H.D. (2009) 'A hybrid approach based on hotelling statistics for automated visual inspection of display blemishes in LCD panels', Expert Systems with Applications, No. 36, pp.13-29.

Davies, E.R. (1998) Automated Visual Inspection, Ch. 19, 2nd ed., Academic Press, London.

Diab, S.L., Basuny, A.M., Ahmed, R. and Hussein, M. (2011) 'Designing a visual inspection system for quality characteristics dimensions', Journal of Mechanical Engineering Research, Vol. 1, No. 1, December, pp.12-23.

Fricout, G., Jeulin, D., Krauth, P-J. and Jacquot, T. (2008) 'Automatic on-line inspection of nonsmooth surface', Wear, Vol. 264, pp.416-421.

Gonzalez, R.C. and Woods, R.E. (2002) Digital Image Processing, Prentice-Hall, Inc., New Jersey.

Hussein, M. and Diab, S.L. (2010a) 'New approaches for on-line visual inspection of products with multiple-characteristics and known tolerances', Int. J. of Rapid Manufacturing, Vol. 1, No. 3, pp.363-375.

Hussein, M. and Diab, S.L. (2010b) 'Visual inspection of products with geometrical quality characteristics of known tolerances', Ain Shams Engineering Journal, Vol. 1, No. 1, pp.79-84.

Hussein, M. and Diab, S.L. (2011) 'Visual inspection of dimensions and positions of geometrical quality characteristics', Int. Journal of Quality and Innovation, Vol. 1, No. 4, pp.326-337.

Kazdin, A.E. (2011) Fundamentals of Digital Image Processing, Oxford University Press, New York, NY, USA, xi 452 pages.

Lee, S. and Vachtsevanos, G. (2002) 'An application of rough set theory to defect detection of automotive glass', Proceedings of the Institution of Mechanical Engineers, Part B, Journal of Engineering Manufacture, Vol. 216, No. 4, pp.637-641.

Leopold, J., Gunther, H. and Leopold, R. (2003) 'New developments in fast 3D-surface quality control', Measurement, Vol. 33, pp.179-187.

Lin, H-D. (2007) 'Automated visual inspection of ripple defects using wavelet characteristic based multivariate statistical approach', Image and Vision Computing, Vol. 25, pp.1785-1801.

Lin, H-D. (2008) 'Tiny surface defect inspection of electronic passive components using discrete cosine transform decomposition and cumulative sum techniques', Image and Vision Computing, Vol. 26, No. 5, pp.603-621.

Lin, H-D. (2009) 'Automated defect inspection of light-emitting diode chips using neural network and statistical approaches', Expert Systems with Applications, Vol. 36, pp.219-226.

Newman, T.S. and Jain, A.K. (1995) 'A survey of automated visual inspection', Computer Vision and Image Understanding, Vol. 61, No. 2, March, pp.231-262.

Sankaranarayanan, R., Nene, B.M., Dinshaw, K., Rajkumar, R., Shastri, S., Wesley, R., Basu, P., Sharma, R., Thara, S., Budukh, A. and Parkin, D.M. (2003) 'Early detection of cervical cancer with visual inspection methods: a summary of completed and on-going studies in India', Salud pública Méx, Vol. 45, January, Suppl. 3, Cuernavaca.

Zurada, J.M. (1992) Introduction to Artificial Neural Systems, West Publishing, Boston.

\section{Websites}

http://www.cognex.com/visual-inspection-systems-visionview.aspx

http://www.cognex.com/support/insight/ 